\title{
Oral hygiene and periodontal conditions in the Chinese patients with aortic aneurysm
}

\author{
Fang Ding ${ }^{1,2}$, Di Wu ${ }^{2}$, Xiao Han², Li-Jian Cheng ${ }^{3}$, Zheng Sun ${ }^{1 *}$ (D) and Ya-Lin Lv ${ }^{2^{*}}$
}

\begin{abstract}
Background: This cross-sectional study aims to evaluate the association of periodontal conditions and oral hygiene habits in the Chinese patients with an aortic aneurysm (AA).

Methods: A questionnaire and periodontal examinations were carried out in the AA patients and non-AA volunteers recruited from the Center for Cardiac Surgery, Beijing Anzhen Hospital, Capital Medical University between August 2011 and June 2016. General information (e.g., height and weight), history of systemic diseases, and oral hygiene habits (e.g., brushing methods and regular oral examinations) were collected in the self-reported survey. Periodontal examinations, such as plaque index and bleeding index, were conducted in all the subjects. The correlation between periodontal indices and AA was further explored using univariate and multivariate analyses.
\end{abstract}

Results: Our analyses revealed that $87.6 \%$ of AA patients have chronic periodontitis, which is significantly higher than that of the non-AA patients (55.8\%). In addition, AA patients demonstrated more severe periodontal damages with $69.3 \%$ moderate and severe periodontitis, compared to only $16.0 \%$ in the non-AA group. Using AA as the dependent variable and all the potential risk factors as covariates (e.g., gender, age, smoking, obesity, diabetes, hypertension, and hyperlipidemia), a logistic regression analysis was performed to show clinical attachment loss (CAL) being an independent risk factor for $\mathrm{AA}(\mathrm{OR}=2.309,95 \% \mathrm{Cl}: 1.623-3.284, p=0.000)$. In comparison with the non-AA patients, more AA patients have poor oral hygiene habits and don't have regular dental appointments for supra-gingival cleaning.

Conclusion: Poor periodontal condition and dental hygiene were identified in the AA patients, suggesting that periodontitis-induced CAL may play a role in AA disease mechanisms.

Keywords: Aortic aneurysm, Cardiovascular disease, Chronic periodontitis, Clinical attachment loss

\section{Background}

Aortic aneurysm (AA), by definition, is local or diffuse aortectasia with at least 1.5 times the expected normal vascular diameter $[1,2]$. AA may occur at any portion of the arterial system, including central arteries (e.g., thoracic aorta and abdominal aorta) and peripheral arteries (e.g., cerebral artery, the artery of extremities and visceral artery) [1]. AA, more commonly seen in the developed countries, is a critical condition with a male predominance and a high mortality rate once ruptures $(\sim 90 \%)[2,3]$. AA is a multifactorial disease. Risk factors

\footnotetext{
*Correspondence: sunzheng12@vip.126.com; Ivyalin@vip.sina.com 'Department of Oral Medicine, Beijing Stomatology Hospital, Capital Medical University, No.4 Tiantan Xili, Dongcheng District, Beijing 100050, China ${ }^{2}$ Department of Stomatology, Beijing Anzhen Hospital, Capital Medical University, No.2 Anzhen Road, Chaoyang District, Beijing 100029, China Full list of author information is available at the end of the article
}

for aneurysmal dilatation include trauma, infection, chronic inflammation (e.g., atherosclerosis), connective tissue disorders (e.g., Marfan syndrome) and environmental factors (e.g., smoking) [4, 5]. Clinically, AA is strongly associated with atherosclerosis, and share similar risk factors [2]. The atheromatous plaque erodes the aortic wall, destroys the arterial media and results in degeneration of elastic fibers $[2,6]$. Infection and chronic inflammation may play a critical role in the initiation and development of AA [2]. Periodontitis is a chronic inflammatory disease caused by pathogenic bacteria and is correlated with cardiovascular diseases, such as atherosclerosis and AA [7]. In addition, periodontal pathogens were identified in the vascular samples from AA patients [8]. Due to the ageing of the population, changes in the lifestyles, and rise of the number of smokers, the incidence of AA in

(c) The Author(s). 2018 Open Access This article is distributed under the terms of the Creative Commons Attribution 4.0 International License (http://creativecommons.org/licenses/by/4.0/), which permits unrestricted use, distribution, and 
China has increased in the past few decades [9]. Thus far, epidemiological studies exploring the association between $\mathrm{AA}$ and periodontal diseases in the Chinese population have never been done. Our cross-sectional study aimed to investigate the periodontal conditions in AA patients, and further explore the correlation between AA and chronic periodontitis in the Chinese population.

\section{Methods}

\section{Study population}

Patients with aortic aneurysm (AA) were diagnosed and recruited from the Center for Cardiac Surgery, Beijing Anzhen Hospital, Capital Medical University between Jan 2012 and June 2016. This study was approved by The Institutional Review Board of Ethics Committee of Beijing Anzhen Hospital, Capital Medical University. All participants received written and oral information prior to giving written consent, and the study was performed in accordance with the Helsinki II declaration. AA was detected and diagnosed by computed tomography (CT) when the aortic diameter was $50 \%$ greater than the expected normal size. Non-AA volunteers were recruited from the health examination center as the control subjects. To diagnose chronic periodontitis, we followed the Classification of Periodontal Diseases and Conditions published by the International Workshop in 1999 [10]. Based on the level of destruction, chronic periodontitis is graded as mild (periodontal pocket depth $\leq 4 \mathrm{~mm}$, clinical attachment loss (CAL) $1-2 \mathrm{~mm}$, and alveolar resorption $<1 / 3$ of root length), moderate (periodontal pocket depth $\leq 6 \mathrm{~mm}$, CAL 3-5 mm, and alveolar resorption $1 / 3-1 / 2$ of root length) and severe (periodontal pocket depth $>6 \mathrm{~mm}$, $\mathrm{CAL} \geq 5 \mathrm{~mm}$, and alveolar resorption $\geq 1 / 2$ of root length) [11]. Patients were excluded if they had periodontal treatments in the past six months and continuous antibiotics applications in the past three months, pseudo-aneurysm, and aortic dissection, or systemic diseases (e.g., malignant tumors, hepatitis and respiratory system disease).

\section{Questionnaire}

A questionnaire was administered on all the subjects to collect general information (e.g., height, weight, education, socioeconomic status, smoking, and alcohol consumption), past medical history, history of chronic periodontitis and periodontal treatments, and dental hygiene habits. All the investigations were conducted under the clinical protocols: 2012001X, 2015011X, and 2017027X approved by the ethics committee of Beijing Anzhen Hospital, Capital Medical University. We have obtained the consent from both AA patients and non-AA volunteers before the investigation.

\section{Periodontal examination}

The periodontal examinations were completed by the same periodontist. Plaque index (PII), bleeding index (BI), probing depth (PD) and CAL were examined and recorded at six sites (mesial-buccal, mid-buccal, distal-buccal, mesial-lingual, mid-lingual, and distal-lingual sites) of the Ramfjord index teeth (i.e., maxillary first molar, maxillary central incisor, maxillary first premolar, mandibular first molar, mandibular central incisor, and mandibular first premolar) [10]. In addition, missing teeth (excluding third molars) were recorded.

\section{Statistical analysis}

The data were collected and analyzed using the single-blind method by SPSS 13.0 software (SPSS Inc., Chicago, IL, USA). The measurements were demonstrated as a mean \pm standard deviation and analyzed using $t$-test. Independent $t$-test was utilized for the inter-group comparison, and variance analysis was used for the comparison of multiple groups. Using AA as the dependable variable and all the potential risk factors (e.g., gender, age, and CAL) as covariates, a logistic regression analysis was performed to determine whether the periodontal infection was one of the risk factors for AA. $p<0.05$ was considered significant.

\section{Results}

\section{Patient characteristics}

In total, 169 AA patients and 156 non-AA patients were included in this study. In the AA group, there were 130 male and 39 female patients, aged from 40 to 72 years (mean age 56.2 years). In the control group, 129 male and 27 female patients were recruited, aged from 40 to 73 years old (mean age $54.8 \pm 5.0$ years). A questionnaire was conducted to collect the background information of the patients, including gender, age, height, weight, missing teeth (Table 1 ) and systemic conditions (Table 2). To avoid confounding factors, we recruited patients with similar a gender ratio and age range. After matching with age and gender, the number of missing teeth was significantly higher in the AA group than in the control group. In addition, the AA group exhibited significantly higher body mass index (BMI) and the number of smokers with lower educational level (Table 1). Compared to the non-AA group, two AA patients had a family history of $\mathrm{AA}$, and more AA patients had histories of coronary heart disease, hypertension, and cerebrovascular disease. In contrast, there was no statistical significance in diabetic history between two groups (Table 2).

\section{Periodontal conditions}

Periodontal clinical indices, namely PLI, PD, BI, and CAL, of the Ramfjord index teeth were examined (Table 3). In addition, ten patients with chronic periodontitis were randomly selected for the self-repeatability tests of PD and CAL. The intra-group coefficients of correlation between PD and CAL reached 0.98 and 0.96, respectively. Compared to the non-AA group, the periodontal clinical 
Table 1 Demographical comparison between AA group and non-AA group

\begin{tabular}{lllllll}
\hline & Gender $($ M:F) & Age & BMI & Missing teeth & Smoking (Yes:No) & Education $(\leq$ nine yrs: $>$ nine yrs $)$ \\
\hline AA $(n=169)$ & $130: 39$ & $56.2 \pm 8.6$ & $25.5 \pm 4.1$ & 1.7 & $99: 70$ & $93: 76$ \\
Non-AA $(n=156)$ & $129: 27$ & $54.8 \pm 5.0$ & $24.7 \pm 3.6$ & 0.5 & $74: 82$ & $35: 121$ \\
$p$ value & 0.196 & 0.076 & $0.047^{*}$ & $0.000^{*}$ & $0.044^{*}$ & $0.000^{*}$ \\
\hline
\end{tabular}

$B M I$ body mass index. Independent $t$-test was performed; ${ }^{*}: p<0.05$

indices were significantly higher in the AA group, suggestive of the association between chronic periodontitis and AA (Table 3).

In addition, the proportion of patients with periodontitis in the AA group was higher than that in the non-AA group. In particular, $87.6 \%$ of the AA patients (148/169) developed periodontitis, while $55.8 \%$ of the non-AA subjects had periodontitis (87/156). Moreover, patients in the AA group developed more severe periodontal damages, with $69.3 \%$ exhibiting moderate and severe periodontitis. In contrast, $16.0 \%$ of the non-AA subjects had moderate and severe periodontitis (Table 4). Using AA as the dependable variable and all the potential risk factors as covariates, the logistic regression analysis revealed that CAL was an independent risk factor $(\mathrm{OR}=2.309,95 \% \mathrm{CI}, 1.623-3.284, p=0.000)$ for AA.

\section{Oral hygiene habits}

Oral hygiene habits were investigated with a self-reported survey, including brushing methods, brushing frequency, brushing time, brushing sites, the average lifespan of a toothbrush, flossing, supra-gingival scaling and regular dental appointments. Compared to the non-AA subjects, more AA patients demonstrated inaccurate brushing method, low brushing frequency and short brushing time without dental flossing habits. Furthermore, fewer AA patients developed a routine for supra-gingival scaling and periodic dental examinations (Table 5). In our cohort, most AA patients exhibited inaccurate oral hygiene habits and lower self-awareness for oral hygiene maintenance.

\section{Discussion}

Dental diseases are correlated with cardiovascular disorders, such as atherosclerosis-induced acute myocardial infarction [12-14]. A potential connection between periodontal diseases and cardiovascular disorders was proposed in the 1990s, followed by multiple studies supporting this hypothesis in the past two decades $[15,16]$. In addition, moderate to severe periodontal diseases have been demonstrated as a significant independent risk factor for peripheral arterial diseases [17]. AA, a cardiovascular disorder, is highly associated with chronic inflammation and infections [2]. Thus far, many studies have indicated that chronic periodontitis contributes to the initiation and progression of AA $[2,7,8]$. Consistent with the previous observations, in the cohort of our current studies, 148 AA patients exhibited chronic periodontitis (87.6\%), which was significantly higher than that in the non-AA group (55.8\%). Compared to the non-AA subjects, AA patients exhibited poorer periodontal health, namely more missing teeth, greater PII and CAL indices and a higher degree of periodontal damages $(69.2 \%$ moderate to severe chronic periodontitis), suggesting chronic periodontitis is a risk factor for AA incidence.

Studies have revealed that periodontal pathogens, herpes simplex virus (HSV), cytomegalovirus (CMV), Epstein-Barr virus (EBV), and Chlamydia pneumoniae may be closely associated with the initiation and progression of AA [18]. Periodontal pathogens, Aggregatibacter actinomycetemcomitans (A. actinomycetemcomitans), were detected in 7.1\% of the AA samples $[19,20]$. Other bacteria species, Porphyromonas gingivalis (P. gingivalis), Treponema denticola, Tannerella forsythia, Prevotella intermedia, and Campylobacter rectus, were also identified in the AA samples [21]. Both $\mathrm{P}$. gingivalis and A. actinomycetemcomitans have been successfully recovered and cultivated in the atherosclerotic tissues [22].

Periodontal pathogens were found in atherosclerotic lesions of the peripheral and central arterial systems [23]. In addition, periodontal pathogens were discovered in the AA samples, suggesting a bacterial invasion of an existing aneurysm by transient bacteremia [23]. Pathogenic microorganisms play a critical role in the initiation and progression of AA, and oral cavity and periodontal pocket may serve as the niche for these gram-negative bacteria [23]. Two hypotheses have been put forward to explain the

Table 2 Past medical history and family history of AA group and non-AA group

\begin{tabular}{|c|c|c|c|c|c|}
\hline & Family history of AA & Coronary heart disease & Hypertension & Cerebrovascular disease & Diabetes \\
\hline AA $(n=169)$ & 2 & 68 & 103 & 22 & 17 \\
\hline Non-AA $(n=156)$ & 0 & 27 & 38 & 2 & 10 \\
\hline$p$ value & & $0.000^{*}$ & $0.000^{*}$ & $0.000^{*}$ & 0.234 \\
\hline
\end{tabular}

Independent $t$-test was performed; $*$ : $p<0.05$ 
Table 3 Comparison of periodontal clinical indices between AA group and non-AA group

\begin{tabular}{lllll}
\hline & PLI & PD $(\mathrm{mm})$ & BI & CAL $(\mathrm{mm})$ \\
\hline AA $(n=169)$ & $2.4 \pm 0.6$ & $3.55 \pm 0.52$ & $3.0 \pm 0.5$ & $3.09 \pm 1.27$ \\
Non-AA $(n=156)$ & $2.0 \pm 0.4$ & $2.29 \pm 0.49$ & $2.1 \pm 0.7$ & $2.25 \pm 1.03$ \\
$p$ value & $0.000^{*}$ & $0.000^{*}$ & $0.000^{*}$ & $0.000^{*}$
\end{tabular}

Independent $t$-test was performed; *: $p<0.05$

etiopathological mechanisms of periodontitis-induced AA. The infection hypothesis proposed that microorganisms contribute to the AA pathogenesis [23]. Pathogenic bacteria from subgingival dental plaques are introduced into the bloodstream through daily activities (e.g., chewing and tooth brushing). Bacteria-induced inflammatory infiltrates then interact with the microenvironment, leading to the production of cytokines and matrix metalloproteinases (MMPs), and degeneration of collagen fibers in the arterial walls $[19,23]^{\cdot}$ The second hypothesis proposed that periodontal pathogens discovered in the aneurysmal tissues were secondary to the short-term bacteremia invading the existing AA [24].

A clinical study was done to examine the prevalence of periodontitis in patients with abdominal AA or arrhythmia in the Japanese population [25]. The results demonstrated that periodontitis had greater impacts on abdominal AA progression, in comparison with other cardiovascular diseases (e.g., arrhythmia) [25]. Compared to the patients with arrhythmia, the AA patients exhibited fewer well-maintained teeth $(14.6 \pm 2.0$ vs. $20.9 \pm 0.7$, $p<0.05)$ and deeper periodontal pockets $(3.01 \pm 0.26$ vs. $2.52 \pm 0.05 \mathrm{~mm}, p<0.05)$ [25]. In addition, in another age- and gender-matched study in the Japanese patients, periodontal bacteria were detected in both abdominal AA and non-AA patients, but deeper periodontal pocket depth was identified in the AA patients [26]. Though with small sample size in these studies, the results indicated a correlation between periodontitis and AA, which is similar to our findings in the current study.

AA and coronary heart diseases share common risk factors, such as age, gender, smoking, diabetes, BMI,

Table 4 Comparison of periodontal status between AA group and non-AA group

\begin{tabular}{llll}
\hline Periodontal status & AA $(n=169)$ & Non-AA $(n=156)$ & $p$ value \\
\hline Healthy & $0(0 \%)$ & $15(9.6 \%)$ & $0.000^{*}$ \\
Gingivitis & $21(12.4 \%)$ & $54(34.6 \%)$ & $0.000^{*}$ \\
Periodontitis & $148(87.6 \%)$ & $87(55.8 \%)$ & $0.000^{*}$ \\
• Mild & $31(18.3 \%)$ & $62(39.7 \%)$ & $0.000^{*}$ \\
• Moderate & $89(52.7 \%)$ & $15(9.6 \%)$ & $0.000^{*}$ \\
• Severe & $28(16.6 \%)$ & $10(6.4 \%)$ & $0.004^{*}$ \\
\hline
\end{tabular}

Independent $t$-test was performed; $*$ : $p<0.05$
Table 5 Comparison of oral hygiene habits between AA group and non-AA group

\begin{tabular}{|c|c|c|c|}
\hline Oral Hygiene Habits & $\begin{array}{l}\mathrm{AA} \\
(n=169)\end{array}$ & $\begin{array}{l}\text { Non-AA } \\
(n=156)\end{array}$ & $p$ value \\
\hline \multicolumn{4}{|l|}{ Brushing methods } \\
\hline • Horizontal & 63 & 16 & $0.000^{*}$ \\
\hline - Vertical & 44 & 96 & $0.000^{*}$ \\
\hline - Mixed & 62 & 44 & 0.103 \\
\hline Brushing frequency & & & $0.001^{*}$ \\
\hline$\cdot<1$ time/day & 82 & 47 & \\
\hline$\cdot \geq 1$ time/day & 87 & 109 & \\
\hline \multicolumn{4}{|l|}{ Brushing time } \\
\hline$\cdot<1 \mathrm{~min} /$ time & 95 & 52 & $0.000^{*}$ \\
\hline - $1-3 \mathrm{~min} /$ time & 58 & 70 & 0.052 \\
\hline$\cdot>3 \mathrm{~min} /$ time & 16 & 34 & $0.002^{*}$ \\
\hline Brushing sites & & & $0.012^{*}$ \\
\hline • Every surface & 76 & 92 & \\
\hline - Only facial surfaces & 93 & 64 & \\
\hline Average lifespan of a toothbrush & & & $0.001^{*}$ \\
\hline - Three months & 30 & 54 & \\
\hline$\cdot>$ Three months & 139 & 102 & \\
\hline Flossing & & & $0.026^{*}$ \\
\hline - Yes & 6 & 15 & \\
\hline • No & 163 & 141 & \\
\hline Supra-gingival scaling & & & $0.000^{*}$ \\
\hline$\cdot \geq 1$ time/year & 9 & 28 & \\
\hline$\cdot<1$ time/year & 160 & 128 & \\
\hline Periodic dental examinations & & & $0.003^{*}$ \\
\hline - Yes & 15 & 32 & \\
\hline - No & 154 & 124 & \\
\hline
\end{tabular}

Independent $t$-test was performed; * $p<0.05$

hypertension, and hyperlipidemia, which can also be confounding factors in the periodontal research [27, 28]. However, the pathogenesis of AA cannot be fully explained by these risk factors. In the current study, we have adjusted these risk factors in the multivariate analysis $(\mathrm{OR}=2.309,95 \% \mathrm{CI}: 1.623-3.284, p=0.000)$, and discovered that CAL is an independent risk factor for AA. Our results indicated the existing periodontal infections can be another risk factor in the development of AA.

Multiple studies have shown the association of periodontal conditions with $\mathrm{AA}[8,20,25]$, but this is the first study investigating the correlation between oral hygiene habits and AA in the Chinese population. Our results indicated that improper oral hygiene habits, such as inaccurate brushing method, no dental flossing, and no regular supra-gingival scaling, are correlated with the incidence of AA. 


\section{Conclusion}

Health-related knowledge and attitude to primary dental care affect the oral hygiene and oral health of an individual, and further influence the systemic health [29]. In this regard, it is essential to establish a complete oral health education program, reinforce oral hygiene maintenance and promote periodic dental care for the general population.

\section{Abbreviations}

AA: Aortic aneurysm; CAL: Clinical attachment loss; CMV: Cytomegalovirus; $\mathrm{CT}$ : Computed tomography; EBV: Epstein-Barr virus; HSV: Herpes simplex virus

\section{Funding}

This study was supported by the National Natural Science Foundation of China (No. 81170282 and 81641159) and the Special Fund for Research on the Relationship between Oral Diseases and Systemic Diseases in the Chinese Stomatological Association (No. CSA-Z2015-05).

\section{Availability of data and materials}

The datasets generated and analyzed during the current study are available from the corresponding author on reasonable request.

\section{Authors' contributions}

FD and DW contributed to the conception and design of the study; $\mathrm{XH}$ and LJC contributed to the acquisition and analysis of data; ZS and YLL contributed to receiving funding and design of the projet; FD wrote the manuscript; All authors reviewed and approved the final version of the manuscript.

\section{Ethics approval and consent to participate}

Ethics Committee of Beijing Anzhen Hospital, Capital Medical University approved this study. Informed consents for publication were obtained from all participants.

\section{Consent for publication}

Not applicable.

\section{Competing interests}

The authors declare that they have no competing interests.

\section{Publisher's Note}

Springer Nature remains neutral with regard to jurisdictional claims in published maps and institutional affiliations.

\section{Author details}

'Department of Oral Medicine, Beijing Stomatology Hospital, Capital Medical University, No.4 Tiantan Xili, Dongcheng District, Beijing 100050, China. ${ }^{2}$ Department of Stomatology, Beijing Anzhen Hospital, Capital Medical University, No.2 Anzhen Road, Chaoyang District, Beijing 100029, China. ${ }^{3}$ Beijing Anzhen Hospital, Capital Medical University, Beijing Institute of Heart, Lung and Blood Vessel Diseases, Beijing Aortic Disease Center, Beijing, China.

Received: 15 December 2017 Accepted: 23 July 2018

Published online: 08 August 2018

\section{References}

1. Gillum RF. Epidemiology of aortic aneurysm in the United States. J Clin Epidemiol. 1995:48(11):1289-98.

2. Sakalihasan $N$, Limet $R$, Defawe $O D$. Abdominal aortic aneurysm. Lancet. 2005;365(9470):1577-89.

3. Dua A, Kuy S, Lee CJ, Upchurch GR Jr, Desai SS. Epidemiology of aortic aneurysm repair in the United States from 2000 to 2010. J Vasc Surg. 2014;59(6):1512-7.

4. Towbin JA, Casey B, Belmont J. The molecular basis of vascular disorders. Am J Hum Genet. 1999:64(3):678-84.

5. Vardulaki KA, Walker NM, Day NE, Duffy SW, Ashton HA, Scott RA. Quantifying the risks of hypertension, age, sex and smoking in patients with abdominal aortic aneurysm. Br J Surg. 2000;87(2):195-200.

6. Lindholt JS, Heegaard NH, Vammen S, Fasting $\mathrm{H}$, Henneberg EW, Heickendorff L. Smoking, but not lipids, lipoprotein(a) and antibodies against oxidised $L D L$, is correlated to the expansion of abdominal aortic aneurysms. Eur J Vasc Endovasc Surg. 2001;21(1):51-6.

7. Bartova J, Sommerova P, Lyuya-Mi Y, Mysak J, Prochazkova J, Duskova J, Janatova T, Podzimek S. Periodontitis as a risk factor of atherosclerosis. J Immunol Res. 2014;2014:636893.

8. Figuero E, Lindahl C, Marin MJ, Renvert S, Herrera D, Ohlsson O, Wetterling T, Sanz M. Quantification of periodontal pathogens in vascular, blood, and subgingival samples from patients with peripheral arterial disease or abdominal aortic aneurysms. J Periodontol. 2014:85(9):1182-93.

9. Cheng SW, Ting AC, Tsang SH. Epidemiology and outcome of aortic aneurysms in Hong Kong. World J Surg. 2003;27(2):241-5.

10. 1999 International International Workshop for a Classification of Periodontal Diseases and Conditions. Papers. Oak Brook, Illinois, October 30-November 2, 1999. Ann Periodontol. 1999;4(1):i, 1-112.

11. Armitage GC. Development of a classification system for periodontal diseases and conditions. Annals of periodontology. 1999;4(1):1-6.

12. Salo JA, Perhoniemi VJ, Lepantalo MJ, Mattila PS. Prognosis of patients over 75 years of age with a ruptured abdominal aortic aneurysm. World J Surg. 1989:13(4):484-6. discussion 486-487

13. Loesche WJ, Schork A, Terpenning MS, Chen YM, Dominguez BL, Grossman $\mathrm{N}$. Assessing the relationship between dental disease and coronary heart disease in elderly U.S. veterans. J Am Dent Assoc. 1998;129(3):301-11.

14. Soto-Barreras U, Olvera-Rubio JO, Loyola-Rodriguez JP, Reyes-Macias JF, Martinez-Martinez RE, Patino-Marin N, Martinez-Castanon GA, AradillasGarcia C, Little JW. Peripheral arterial disease associated with caries and periodontal disease. J Periodontol. 2013:84(4):486-94.

15. Mattila KJ, Valle MS, Nieminen MS, Valtonen W, Hietaniemi KL. Dental infections and coronary atherosclerosis. Atherosclerosis. 1993;103(2):205-11.

16. Mattila KJ. Dental infections as a risk factor for acute myocardial infarction. Eur Heart J. 1993;14(Suppl K):51-3.

17. Mendez MV, Scott T, LaMorte W, Vokonas P, Menzoian JO, Garcia R. An association between periodontal disease and peripheral vascular disease. Am J Surg. 1998;176(2):153-7.

18. Ismail A, Khosravi $\mathrm{H}$, Olson $\mathrm{H}$. The role of infection in atherosclerosis and coronary artery disease: a new therapeutic target. Heart Dis. 1999;1(4):233-40.

19. Marques da Silva R, Lingaas PS, Geiran O, Tronstad L, Olsen I. Multiple bacteria in aortic aneurysms. J Vasc Surg. 2003;38(6):1384-9.

20. Marques da Silva R, Caugant DA, Lingaas PS, Geiran O, Tronstad L, Olsen I. Detection of Actinobacillus actinomycetemcomitans but not bacteria of the red complex in aortic aneurysms by multiplex polymerase chain reaction. J Periodontol. 2005;76(4):590-4.

21. Kurihara $N$, Inoue $Y$, Iwai $T$, Umeda M, Huang $Y$, Ishikawa I. Detection and localization of periodontopathic bacteria in abdominal aortic aneurysms. Eur J Vasc Endovasc Surg. 2004;28(5):553-8.

22. Rafferty B, Jonsson D, Kalachikov S, Demmer RT, Nowygrod R, Elkind MS, Bush H Jr, Kozarov E. Impact of monocytic cells on recovery of uncultivable bacteria from atherosclerotic lesions. J Intern Med. 2011;270(3):273-80.

23. Paraskevas KI, Mikhailidis DP, Giannoukas AD. Periodontitis and abdominal aortic aneurysms: a random association or a pathogenetic link? Int Angiol. 2009;28(6):431-3.

24. Parahitiyawa NB, Jin LJ, Leung WK, Yam WC, Samaranayake LP. Microbiology of odontogenic bacteremia: beyond endocarditis. Clin Microbiol Rev. 2009; 22(1):46-64. Table of Contents

25. Suzuki J, Aoyama N, Aoki M, Tada Y, Wakayama K, Akazawa H, Shigematsu K, Hoshina K, Izumi Y, Komuro I, Miyata T, Hirata Y, Isobe M. High incidence of periodontitis in Japanese patients with abdominal aortic aneurysm. Int Heart J. 2014:55(3):268-70.

26. Suzuki J, Aoyama N, Aoki M, Tada Y, Wakayama K, Akazawa H, Shigematsu K, Hoshina K, Izumi Y, Komuro I, Miyata T, Hirata Y, Isobe M. Incidence of periodontitis in Japanese patients with cardiovascular diseases: a comparison between abdominal aortic aneurysm and arrhythmia. Heart Vessel. 2015;30(4):498-502.

27. Scannapieco FA, Cantos A. Oral inflammation and infection, and chronic medical diseases: implications for the elderly. Periodontol 2000. 2016;72(1):153-75.

28. Genco RJ, Borgnakke WS. Risk factors for periodontal disease. Periodontol 2000. 2013:62(1):59-94.

29. Redfern J. Smart health and innovation: facilitating health-related behaviour change. Proc Nutr Soc. 2017;76(3):328-32 